TRANSACTIONS OF THE

AMERICAN MATHEMATICAL SOCIETY

Volume 349, Number 7, July 1997, Pages 2845-2870

S $0002-9947(97) 01767-4$

\title{
ON THE DENJOY RANK, THE KECHRIS-WOODIN RANK AND THE ZALCWASSER RANK
}

\author{
HASEO KI
}

\begin{abstract}
We show that the Denjoy rank and the Zalcwasser rank are incomparable. We construct for any countable ordinal $\alpha$ differentiable functions $f$ and $g$ such that the Zalcwasser rank and the Kechris-Woodin rank of $f$ are $\alpha+1$ but the Denjoy rank of $f$ is 2 and the Denjoy rank and the KechrisWoodin rank of $g$ are $\alpha+1$ but the Zalcwasser rank of $g$ is 1 . We then derive a theorem that shows the surprising behavior of the Denjoy rank, the Kechris-Woodin rank and the Zalcwasser rank.
\end{abstract}

\section{INTRODUCTION}

Kechris and Woodin $[\mathrm{KW}]$, Ajtai and Kechris [AK], Ramsamujh [Ra] and Ki [Ki] conjectured that the Denjoy rank is greater than or equal to the Zalcwasser rank. In this article, we prove that this conjecture is false.

It is natural to ask how we can recover a primitive from its derivative. This is known as Newton's problem. In 1912, Denjoy solved the general problem of the primitive via the Denjoy process, which consists of a countable iteration of Lebesgue integrations and summations of series. From the Denjoy process, one can define a rank on the set of differentiable functions. It is called the Denjoy rank. The Denjoy rank is unbounded below the first uncountable ordinal.

Zalcwasser $[\mathrm{Za}]$, and Gillespie and Hurwitz $[\mathrm{GH}]$ introduced another rank that measures how far from being uniform is the pointwise convergence of sequences of continuous functions on the unit interval. For each continuous function on the unit circle, we can consider its Fourier series and apply the Zalcwasser rank to the sequence of the partial Fourier sums. Ajtai and Kechris [AK] have shown, in a nonconstructive way, that on the set of all continuous functions with convergent Fourier series, the Zalcwasser rank is a $\Pi_{1}^{\mathbf{1}}$-norm which is unbounded below $\omega_{1}$. Also, Ki [Ki] has, constructively, proven the unboundedness of the Zalcwasser rank on the set. It is well known that for each differentiable function $f$ on the unit circle, the Fourier series of $f$ converges pointwise. So every differentiable function on the unit circle has a countable ordinal Zalcwasser rank, and we will consider the Zalcwasser rank on the set of all differentiable functions on the unit circle. Ajtai and Kechris [AK] have demonstrated, non-constructively, the unboundedness of the Zalcwasser rank on the set of all differentiable functions on the unit circle.

Received by the editors April 13, 1995 and, in revised form, January 18, 1996.

1991 Mathematics Subject Classification. Primary 04A15, 26A21; Secondary 42 A20.

Key words and phrases. Denjoy rank, descriptive set theory, Fourier series, Kechris-Woodin rank, Zalcwasser rank.

Partially supported by GARC-KOSEF. 
In addition to the above ranks, Kechris and Woodin [KW] developed a rank that measures how far the derivative is from being continuous. They have shown that on the set of all differentiable functions, the Kechris-Woodin rank is a $\Pi_{1}^{1}$-norm and one can construct arbitrarily high ranked differentiable functions with bounded derivatives. But Mazurkiewicz [Ma] has shown that the set of all differentiable functions is $\Pi_{1}^{1}$-complete, in particular, $\Pi_{1}^{1}$ but non-Borel. Then by a fundamental theorem on $\Pi_{1}^{1}$-norms, the Kechris-Woodin rank is unbounded below the first uncountable ordinal.

As we have seen, we can consider three different ranks on the set of all differentiable functions which have their own purposes. Roughly speaking, small ranks mean the function is well behaved and big ranks imply bad behavior. A basic question is to compare these three different ranks. Ramsamujh [Ra] has shown that for any differentiable function $f$, the Kechris-Woodin rank of $f$ is greater than or equal to the Denjoy rank of $f$. Recently, it was shown in [Ki] that the KechrisWoodin rank is greater than or equal to the Zalcwasser rank. Therefore, only one such question is left to ask, whether the Denjoy rank and the Zalcwasser rank are comparable. It has been believed until now that the Denjoy rank is always greater than or equal to the Zalcwasser rank, and this was supported by a fair amount of evidence. For example, for a differentiable function $f$ with bounded derivative, the Denjoy rank of $f$ is 1 (i.e., $f^{\prime}$ is integrable), and then so is the Zalcwasser rank of $f$. However, surprisingly, the conjecture fails: for any countable ordinal $\alpha$, one can construct two differentiable functions $f$ and $g$ such that the Zalcwasser rank and the Kechris-Woodin rank of $f$ are $\alpha+1$ but the Denjoy rank of $f$ is 2 , while the Denjoy rank and Kechris-Woodin rank of $g$ are $\alpha+1$ but the Zalcwasser rank of $f$ is 1. Therefore, the Denjoy rank and the Zalcwasser rank are not comparable.

By our result and a fundamental theorem on $\Pi_{1}^{1}$-norms, we then reprove that the set of all differentiable functions and the set of all continuous functions with convergent Fourier series are $\Pi_{1}^{1}$ but non-Borel.

Combining our result and the Kechris and Woodin theorem, which says for any nonzero countable ordinal $\alpha$ there exists a differentiable function with bounded derivative whose Kechris-Woodin rank is $\alpha$, we have an additional theorem. Namely, for any countable ordinal $\alpha$ all nonzero countable ordinals $\beta$ and $\gamma$ with $\alpha, \beta<\gamma$, there exists a differentiable function $f$ such that the Zalcwasser rank of $f$ is $\alpha+1$, the Denjoy rank of $f$ is $\beta+1$ and the Kechris-Woodin rank of $f$ is $\gamma$. This theorem shows that there are differentiable functions with arbitrary (successor) Denjoy rank $>1$, arbitrary (successor) Zalcwasser rank, and arbitrary Kechris-Woodin rank bigger than the other two.

\section{DEFINITIONS AND BACKGROUND}

Let $\mathbb{N}=\{1,2,3, \cdots\}$ be the set of positive integers and $\mathbb{N}^{\mathbb{N}}$ the Polish space with the usual product topology, where $\mathbb{N}$ is given the discrete topology. Let $\mathbb{R}$ be the set of real numbers. Let $f$ be a real valued function on a space $X$. We then define

$$
\|f\|=\sup \{|f(x)|: x \in X\} .
$$

We denote by $\left\langle f_{n}\right\rangle$ a sequence of functions. We use the standard notations $o$ and $O$ as folllows. For real valued functions $\left\langle f_{n}\right\rangle$ and $\left\langle g_{n}\right\rangle$ on a space $X, f_{n}=g_{n}+o(1)$, if for each $x \in X, \lim _{n \rightarrow \infty} f_{n}(x)-g_{n}(x)=0$ and $f_{n}=O\left(g_{n}\right)$, if for some positive constant $C,\left\|f_{n}\right\| \leq C\left\|g_{n}\right\|$ for all $n \in \mathbb{N}$. Let $\mathbb{T}$ denote the unit circle. We consider $\mathbb{T}$ as $[0,2 \pi]$, identifying 0 with $2 \pi$. Let $C(\mathbb{T})$ denote the set of continuous functions 
on $\mathbb{T}$ with the sup metric induced by $\|\cdot\| . C(\mathbb{T})$ can also be considered as the space of all continuous $2 \pi$-periodic functions on $\mathbb{R}$ by viewing $\mathbb{T}$ as $\mathbb{R} / 2 \pi \mathbb{Z}$. We denote by $D(\mathbb{T})$ the set of differentiable functions on $\mathbb{T}$. We associate to each $f \in C(\mathbb{T})$ its Fourier series $S[f] \sim \sum_{n=-\infty}^{\infty} \hat{f}(n) e^{i n x}$ where $\hat{f}(n)=\frac{1}{2 \pi} \int_{0}^{2 \pi} f(t) e^{-i n t} d t$. Let

$$
S_{n}(f, t)=\sum_{k=-n}^{n} \hat{f}(k) e^{i k t}
$$

be the $n$th partial sum of the Fourier series of $f$. We say "the Fourier series of $f$ converges at a point $t \in \mathbb{T}$ " if the sequence $\left\langle S_{n}(f, t)\right\rangle_{n \in \mathbb{N}}$ converges. We denote by $E C$ the set of all continuous functions on the unit circle with convergent Fourier series. According to a standard theorem $[\mathrm{Ka}]$, if the Fourier series of $f$ at $t$ converges, then it must converge to $f(t)$. Hence,

$$
\begin{aligned}
E C & =\left\{f \in C(\mathbb{T}): \text { for all } t \in[0,2 \pi],\left\langle S_{n}(f, t)\right\rangle_{n \in \mathbb{N}} \text { converges }\right\} \\
& =\left\{f \in C(\mathbb{T}): \text { for all } t \in[0,2 \pi], f(t)=\lim _{n \rightarrow \infty} S_{n}(f, t)\right\} .
\end{aligned}
$$

Let $\mathcal{K}(\mathbb{T})$ denote the set of all closed sets in $\mathbb{T} . \mathcal{K}(\mathbb{T})$ is a Polish space with the Hausdorff metric. We denote by $\mathcal{K}_{\aleph_{0}}(\mathbb{T})$ the set of all countable closed sets in $\mathbb{T}$.

A subset $A$ of a Polish space $X$ is called $\Pi_{1}^{1}$, if there is a Borel function $f$ from $\mathbb{N}^{\mathbb{N}}$ to $X$ such that the image under $f$ of $\mathbb{N}^{\mathbb{N}}$ is $X-A$, i.e., $f\left(\mathbb{N}^{\mathbb{N}}\right)=X-A$. Thus a $\Pi_{1}^{1}$ set is coanalytic. By a standard method of descriptive set theory, one can prove that $E C, D(\mathbb{T})$, and $\mathcal{K}_{\aleph_{0}}(\mathbb{T})$ are $\Pi_{1}^{1}$. A $\Pi_{1}^{1}$ subset $A$ of $X$ is called complete, if for any Polish space $Y$ and any $\Pi_{1}^{1}$ subset $B$ of $Y$, there is a Borel function $f$ from $Y$ to $X$ such that the preimage under $f$ of $A$ is $B$, i.e., $B=f^{-1}(A)$. It is easy to see that all complete $\Pi_{1}^{1}$ sets are non-Borel.

We define a $\Pi_{1}^{1}$-norm on a $\Pi_{1}^{1}$ set. A norm on a set $P$ is any function $\varphi$ taking $P$ into the ordinals. $\varphi$ is regular if $\varphi$ maps $P$ onto some ordinal $\lambda$. Two norms $\varphi$ and $\psi$ on $P$ are equivalent if $\varphi(x) \leq \varphi(y)$ if and only if $\psi(x) \leq \psi(y)$. Every norm is equivalent to a unique regular norm. Given a Polish space $X$ and a $\Pi_{1}^{1}$ subset $P$ of $X$, we say that a norm $\varphi: P \rightarrow$ Ordinals is a $\Pi_{1}^{1}$-norm if there are $\Pi_{1}^{\mathbf{1}}$ subsets $R$ and $Q$ of $X \times X$ such that

$$
\begin{aligned}
y \in P \text { implies } & {[x \in P \text { and } \varphi(x) \leq \varphi(y)} \\
& \text { if and only if }(x, y) \notin R \\
& \text { if and only if }(x, y) \in Q] .
\end{aligned}
$$

If a subset $A$ of a Polish space and its complement are both $\Pi_{1}^{\mathbf{1}}$, then $A$ is Borel by a theorem of Suslin (see $[\mathrm{Mo}]$ or $[\mathrm{Ke}]$ ). Hence in $(*)$, we see that in a uniform manner for $y \in P$, the set $\{x \in P: \varphi(x) \leq \varphi(y)\}$ is $\Pi_{1}^{1}((x, y) \in Q)$ and the complement of a $\Pi_{1}^{1}$ set $((x, y) \notin R)$, hence a Borel set. One of the basic facts is that every $\Pi_{1}^{1}$ subset $P$ admits a $\Pi_{1}^{1}$-norm $\varphi: P \rightarrow \omega_{1}$ (see $[\mathrm{Mo}]$ or $[\mathrm{Ke}]$ ). Hence it is very natural to look for a canonical norm on $\Pi_{1}^{1}$ sets that arise in analysis, topology, etc. We will introduce $\Pi_{1}^{1}$-norms on the set of all countable closed sets, the set of differentiable functions and the set of continuous functions with everywhere convergent Fourier series. From rank theory, we have the following fundamental theorem (see [Mo] or $[\mathrm{Ke}])$.

Boundedness Principle. Let $X$ be a Polish space. Let $P$ be a $\Pi_{1}^{1}$ subset of $X$ and $\varphi: P \rightarrow \omega_{1}$ be a $\Pi_{1}^{1}$-norm on $P$. Then $P$ is Borel if and only if $\varphi$ is bounded below $\omega_{1}$. 
Thus, one can prove that a $\Pi_{1}^{1}$ set $P$ is $\Pi_{1}^{1}$ non-Borel by showing that some $\Pi_{1}^{1}$-norm on $P$ is unbounded below $\omega_{1}$.

It is known that $D(\mathbb{T})$ and $E C$ are $\Pi_{1}^{1}$ but non-Borel (see $[\mathrm{KW}]$ and $[\mathrm{Ma}]$ for $D(\mathbb{T})$ and $[\mathrm{AK}]$ and $[\mathrm{Ki}]$ for $E C)$. By the Boundedness Principle and our constructions, we will reprove the non-Borelness of $D(\mathbb{T})$ and $E C$.

\section{The Denjoy RANK}

We introduce the Denjoy rank. Let $g$ be a measurable function on $\mathbb{T}$, and let $P$ be a closed subset of $\mathbb{T}$. We define the set of all singular points of $g$ over $P$ by

$$
\begin{aligned}
S(g, P)=\{x \in P: & \text { for any open interval } I \text { with } x \in I, \\
& g \text { is not Lebesgue integrable on } I \cap P\} \\
=\{x \in P: & \text { for any open interval } I \text { with } x \in I, \\
& \left.\int_{I \cap P}|g(t)| d t=\infty\right\} .
\end{aligned}
$$

Let $f \in C(\mathbb{T})$. Let $\left\langle\left(a_{n}, b_{n}\right)\right\rangle$ be an enumeration of the connected components of the complement of $P$ in $\mathbb{T}$. We define the set of divergence points of $f$ over $P$ by

$$
\begin{gathered}
D(f, P)=\{x \in P: \text { for every open interval } I \text { with } x \in I, \\
\left.\qquad \sum_{I}\left|f\left(b_{n}\right)-f\left(a_{n}\right)\right| \text { diverges }\right\} .
\end{gathered}
$$

Here $\sum_{I}$ indicates that the sum is to be taken over all the intervals $\left(a_{n}, b_{n}\right)$ which are contained in $I$. For $f \in D(\mathbb{T})$ and each closed subset $P$ of $\mathbb{T}$, we define the $D J$-derived set of $P$ by

$$
\partial_{f}^{D J}(P)=S\left(f^{\prime}, P\right) \cup D(f, P) .
$$

We note that for $f \in D(\mathbb{T}), S\left(f^{\prime}, P\right)$ is well-defined since $f^{\prime}$ is measurable. Also, $\partial_{f}^{D J}(P)$ is a closed subset of $\mathbb{T}$. We then define the transfinite sequence $\left\langle\partial_{f}^{D J}(P, \alpha)\right\rangle_{\alpha<\omega_{1}}$. By transfinite induction, we let $\partial_{f}^{D J}(P, 0)=P$ and

$$
\partial_{f}^{D J}(P, \alpha+1)=\partial_{f}^{D J}\left(\partial_{f}^{D J}(P, \alpha)\right) .
$$

For $\lambda$ a limit ordinal,

$$
\partial_{f}^{D J}(P, \lambda)=\bigcap_{\alpha<\lambda} \partial_{f}^{D J}(P, \alpha)
$$

For each $f \in D(\mathbb{T})$, let $|f|_{D J}$ be the least ordinal $\alpha$ for which $\partial_{f}^{D J}(\mathbb{T}, \alpha)=\emptyset$. Note that for each $f \in D(\mathbb{T}),|f|_{D J}$ is countable. For $f \in D(\mathbb{T})$, it is known that $|f|_{D J}=1$ if and only if $f^{\prime}$ is integrable. We give an example whose Denjoy rank is not 1 . We define the function

$$
s(x)= \begin{cases}x^{2} \sin 1 / x^{2}, & \text { if } x \neq 0 \\ 0, & \text { otherwise }\end{cases}
$$

Let $d$ be the function obtained by reflecting the graph of $s \uparrow[0, b]$ in the line $x=\pi$ and by joining the two maxima at $b$ and $2 \pi-b$ by a straight line. Then $d \in D(\mathbb{T})$ and easily the Denjoy rank of $d$ is 2 .

We will use the function $d$ to construct arbitrarily high Denjoy ranks with Zalcwasser ranks being 1 .

For a more detailed description of the Denjoy process, we refer to $[\mathrm{Br}]$. 


\section{The KeCHRIS-WOODIN RANK}

We define a $\Pi_{1}^{1}$-norm on $D(\mathbb{T})$, which we refer to as the Kechris-Woodin rank. Fix $f \in C(\mathbb{T})$ and $\varepsilon>0$. For each closed subset $P$ of $\mathbb{T}$, we define the $K W$-derived set of $P$ by

$$
\begin{aligned}
\partial_{f, \varepsilon}^{K W}(P)=\{x \in P: & \text { for any open neighborhood } U \text { of } x, \\
& \text { there exist closed intervals }[a, b],[c, d] \subseteq U \text { such that }
\end{aligned}
$$

$$
\left.[a, b] \cap[c, d] \cap P \neq \emptyset \text { and }\left|\frac{f(b)-f(a)}{b-a}-\frac{f(d)-f(c)}{d-c}\right| \geq \varepsilon\right\} .
$$

$\partial_{f, \varepsilon}^{K W}(P)$ consists of all $\varepsilon$-badly behaved points of $P$ in terms of the derivative of $f$. Clearly $\partial_{f, \varepsilon}^{K W}(P)$ is closed. We can then define the sequence $\left\langle\partial_{f, \varepsilon}^{K W}(P, \alpha)\right\rangle_{\alpha<\omega_{1}}$ by transfinite induction. Let $\partial_{f, \varepsilon}^{K W}(P, 0)=P$ and

$$
\partial_{f, \varepsilon}^{K W}(P, \alpha+1)=\partial_{f, \varepsilon}^{K W}\left(\partial_{f, \varepsilon}^{K W}(P, \alpha)\right) .
$$

For $\lambda$ a limit ordinal,

$$
\partial_{f, \varepsilon}^{K W}(P, \lambda)=\bigcap_{\alpha<\lambda} \partial_{f, \varepsilon}^{K W}(P, \alpha)
$$

Note that

$$
\bigcup_{\varepsilon>0} \partial_{f, \varepsilon}^{K W}(P, \alpha)=\bigcup_{n \in \mathbb{N}} \partial_{f, \frac{1}{n}}^{K W}(P, \alpha)
$$

Define the sequence $\left\langle\partial_{f}^{K W}(P, \alpha)\right\rangle_{\alpha<\omega_{1}}$ by setting

$$
\partial_{f}^{K W}(P, \alpha)=\bigcup_{n \in \mathbb{N}} \partial_{f, \frac{1}{n}}^{K W}(P, \alpha)
$$

We refer to $[\mathrm{KW}]$ for the following fact.

Fact 1. $f \in D(\mathbb{T})$ if and only if for some $\alpha<\omega_{1}, \partial_{f}^{K W}(\mathbb{T}, \alpha)=\emptyset$.

Using this fact, we can define the Kechris-Woodin rank on $D(\mathbb{T})$. For each $f \in D(\mathbb{T})$, let $|f|_{K W}=$ the least ordinal $\alpha$ for which $\partial_{f}^{K W}(\mathbb{T}, \alpha)=\emptyset$. We let $b_{1} D(\mathbb{T})$ be the set of all functions whose derivatives are bounded in absolute value by 1 . It is easy to show that $b_{1} D(\mathbb{T})$ is $\Pi_{1}^{1}$. We also have the following theorem of Kechris and Woodin:

Theorem KW $[\mathrm{KW}]$. For any nonzero ordinal $\alpha<\omega_{1}$, there exists a function $f$ in $b_{1} D(\mathbb{T})$ such that the Kechris-Woodin rank of $f$ is $\alpha$.

By a simple argument, for each $f \in D(\mathbb{T})$, the Kechris-Woodin rank of $f$ is 1 if and only if $f^{\prime}$ is continuous. Also the Kechris-Woodin rank of $d$ is 2, like the Denjoy rank. Clearly, the Denjoy rank of $f$ with continuous $f^{\prime}$ is 1, i.e., if $|f|_{K W}=1$, so is the Denjoy rank of $f$. In fact, Ramsamujh has shown that it is true in general.

Theorem $\mathbf{R}[\mathrm{Ra}]$. For any differentiable function $f$, the Kechris-Woodin rank of $f$ is greater than or equal to the Denjoy rank of $f$.

The following fact appears in $[\mathrm{KW}]$.

Fact 2. $|\cdot|_{K W}: D(\mathbb{T}) \rightarrow \omega_{1}$ is a $\Pi_{1}^{1}$-norm. 
Kechris and Woodin $[\mathrm{KW}]$ have constructed for any countable ordinal $\alpha$ a differentiable function $f$ in $b_{1} D(\mathbb{T})$ whose Kechris-Woodin rank is $\alpha$. By this fact, Fact 2 and the Boundedness Principle, the sets $D(\mathbb{T})$ and $b_{1} D(\mathbb{T})$ are $\Pi_{1}^{1}$ non-Borel subsets of $C(\mathbb{T})$. In fact, Mazurkiewicz [Ma] has shown that $D(\mathbb{T})$ is $\Pi_{1}^{1}$-complete.

\section{THE ZALCWASSER RANK}

We will give a rank on the set $E C$ of all continuous functions with everywhere convergent Fourier series. Let $f \in C(\mathbb{T})$, let $P \subseteq \mathbb{T}$ be a closed set, and let $x \in P$. We define the value of the oscillation function of $f$ on $P$ at $x$ as follows.

$\omega(x, f, P)=\inf _{\delta>0} \inf _{p \in \mathbb{N}} \sup \left\{\left|S_{m}(f, y)-S_{n}(f, y)\right|: m, n \geq p, y \in P\right.$ and $\left.|x-y|<\delta\right\}$.

Thus the oscillation function of $f$ on $P$ measures how bad the uniform convergence of the Fourier series of $f$ near $x$ is on $P$. For each $f \in C(\mathbb{T})$ and each $\varepsilon>0$, define the $Z$-derived set of $P$ by

$$
\partial_{f, \varepsilon}^{Z}(P)=\{x \in P: \omega(x, f, P) \geq \varepsilon\} .
$$

Note that $\partial_{f, \varepsilon}^{Z}(P)$ is a closed subset of $\mathbb{T}$. As before, we define $\left\langle\partial_{f, \varepsilon}^{Z}(P, \alpha)\right\rangle_{\alpha<\omega_{1}}$ by transfinite induction. We then define a transfinite sequence $\left\langle\partial_{f}^{Z}(P, \alpha)\right\rangle_{\alpha<\omega_{1}}$.

We refer to $[\mathrm{AK}]$ for the following two facts.

Fact 3. $f \in E C$ if and only if for some $\alpha<\omega_{1}, \partial_{f}^{Z}(\mathbb{T}, \alpha)=\emptyset$.

Using this fact, we define the Zalcwasser rank as follows. For each $f \in E C$, let $|f|_{Z}=$ the least ordinal $\alpha$ for which $\partial_{f}^{Z}(\mathbb{T}, \alpha)=\emptyset$.

Fact 4. $|\cdot|_{Z}: E C \rightarrow \omega_{1}$ is a $\Pi_{1}^{1}$-norm.

It is not hard to demonstrate that for each $f \in E C$, the Zalcwasser rank of $f$ is 1 if and only if the Fourier series of $f$ converges uniformly. The function $d$ may seem to be a good candidate to have Zalcwasser rank greater than 1 , but we will see that in fact, the Zalcwasser rank of $d$ is just 1 . However, the author [Ki] has constructed arbitrarily high Zalcwasser ranks in $E C$.

Theorem [Ki]. One can construct for each nonzero countable ordinal $\alpha$ a function in EC whose Zalcwasser rank exceeds $\alpha$.

Also the natural question was asked in $[\mathrm{AK}],[\mathrm{KW}]$ and $[\mathrm{Ra}]$ whether or not the Kechris-Woodin rank is greater than or equal to the Zalcwasser rank. We have obtained a positive answer to this.

Theorem K [Ki]. For any differentiable function on the unit circle, the KechrisWoodin rank of $f$ is greater than or equal to the Zalcwasser rank.

Note that by a standard fact, every differentiable function has an everywhere convergent Fourier series, i.e., $D(\mathbb{T}) \subseteq E C$. Ajtai and Kechris $[\mathrm{AK}]$ have shown that no Borel set $B$ exists with $D(\mathbb{T}) \subseteq B \subseteq E C$. This implies the following fact:

Fact 5. For each $\alpha<\omega_{1}$, there is a differentiable function $f$ such that $|f|_{Z} \geq \alpha$.

In particular, by these facts and the Boundedness Principle, $D(\mathbb{T})$ is $\Pi_{1}^{1}$ but non-Borel. In fact, Ajtai and Kechris [AK] have shown that $E C$ is $\Pi_{1}^{1}$-complete. 


\section{The Cantor-Bendixon Rank}

We define a rank on the set $\mathcal{K}_{\aleph_{0}}(\mathbb{T})$ of all countable closed sets, known as the Cantor-Bendixon rank. Let $P$ be a closed subset of $\mathbb{T}$. We denote by $\partial^{C B}(P)$ the set of all limit points of $P$, i.e.,

$$
\partial^{C B}(P)=\{x \in P: x \text { is a limit point of } P\} .
$$

Clearly this set $\partial^{C B}(P)$ is a closed subset of $\mathbb{T}$. We define a transfinite sequence $\left\langle\partial^{C B}(P, \alpha)\right\rangle_{\alpha<\omega_{1}}$. By transfinite induction, we let $\partial^{C B}(P, 0)=P$ and

$$
\partial^{C B}(P, \alpha+1)=\partial^{C B}\left(\partial^{C B}(P, \alpha)\right) .
$$

For $\lambda$ a limit ordinal,

$$
\partial^{C B}(P, \lambda)=\bigcap_{\alpha<\lambda} \partial^{C B}(P, \alpha) .
$$

A basic fact is that for each closed subset $P$ of $\mathbb{T}, P$ is in $\mathcal{K}_{\aleph_{0}}(\mathbb{T})$ if and only if for some $\alpha<\omega_{1}, \alpha$ many iterations of $P$ end in the empty set, i.e., $\partial^{C B}(P, \alpha)=\emptyset$. This allows us to define a rank from $\mathcal{K}_{\aleph_{0}}(\mathbb{T})$ to $\omega_{1}$. For each $P \in \mathcal{K}_{\aleph_{0}}(\mathbb{T}),|P|_{C B}=$ the least countable ordinal $\alpha$ for which $\partial^{C B}(P, \alpha)=\emptyset$. It is not hard to show the following:

Fact 6. $|\cdot|_{C B}: \mathcal{K}_{\aleph_{0}}(\mathbb{T}) \rightarrow \omega_{1}$ is a $\Pi_{1}^{1}-$ norm.

We will use the following remark in the constructions of arbitrarily high ranked differentiable functions.

Remark 7. Let $f$ be in $D(\mathbb{T})$. Suppose $\partial_{f}^{D J}(\mathbb{T}, 1), \partial_{f}^{K W}(\mathbb{T}, 1)$, and $\partial_{f}^{Z}(\mathbb{T}, 1)$ are countable. Then for any ordinal $\gamma$ and any $\delta>0$,

$$
\begin{aligned}
& \partial_{f}^{D J}(\mathbb{T}, \gamma) \subseteq \partial^{C B}\left(\partial_{f}^{D J}(\mathbb{T}, 1), \gamma-1\right), \\
& \partial_{f, \delta}^{K W}(\mathbb{T}, \gamma) \subseteq \partial^{C B}\left(\partial_{f, \delta}^{K W}(\mathbb{T}, 1), \gamma-1\right), \\
& \partial_{f, \delta}^{Z}(\mathbb{T}, \gamma) \subseteq \partial^{C B}\left(\partial_{f, \delta}^{Z}(\mathbb{T}, 1), \gamma-1\right),
\end{aligned}
$$

where $\gamma-1=\gamma$ if $\gamma$ is a limit ordinal.

\section{Construction of FUnCtions on $D(\mathbb{T})$ HaVing arbitrarily bad Denjoy RANK WITH ZALCWASSER RANK 1}

Ramsamujh [Ra] has constructed a differentiable function whose Denjoy rank is 2 and whose Zalcwasser rank is 1 . This seems to be the best known result. However, we will build differentiable functions whose Denjoy ranks are unbounded below $\omega_{1}$ but whose Zalcwasser ranks are only 1 . This construction strongly suggests that the Zalcwasser rank is less than or equal to the Denjoy rank in general (note that the next section will contain the converse of this result). For our construction, we will essentially follow Denjoy's method, which establishes that the Denjoy rank is unbounded below the first uncountable ordinal, but we shall need a basic fact from Fourier analysis to prove the uniform convergence of the Fourier series of a given function.

We first give some background in Fourier analysis. Let $f$ be a function on $\mathbb{T}$ and $\delta>0$. We define the modulus of continuity of $f$ as follows:

$$
\omega(\delta, f)=\sup \{|f(x)-f(y)|: x, y \in \mathbb{T} \text { and }|x-y| \leq \delta\} .
$$


We need the following test to prove the uniform convergence of the Fourier series of a given function.

The Dini-Lipschitz test. Suppose $f$ is continuous and its modulus of continuity $\omega(\delta, f)$ satisfies the condition $\omega(\delta, f) \log \delta \rightarrow 0$ as $\delta \rightarrow 0$. Then the Fourier series of $f$ converges uniformly.

By this test, we can avoid calculating the whole Fourier series of a given function whose Fourier series is supposed to converge uniformly. Namely, in order to show the uniform convergence of the Fourier series of a given function, it suffices to deal with the basic functional property of the function.

Proposition 8. The Zalcwasser rank of the function $d$ is 1 .

Proof of Proposition 8. By virtue of the Dini-Lipschitz test, it suffices to show that $\omega(\delta, s) \log \delta \rightarrow 0$ as $\delta \rightarrow 0$, where

$$
s(x)= \begin{cases}x^{2} \sin 1 / x^{2}, & \text { if } x \neq 0 \\ 0, & \text { otherwise. }\end{cases}
$$

By the definition of the modulus of continuity, we easily get

$$
\begin{aligned}
\omega(\delta, s) & \leq \sup _{\left|x_{1}-x_{2}\right|<\delta}\left|\left(x_{1}^{2}-x_{2}^{2}\right) \sin \frac{1}{x_{1}^{2}}\right|+\sup _{\left|x_{1}-x_{2}\right|<\delta} x_{2}^{2}\left|\sin \frac{1}{x_{1}^{2}}-\sin \frac{1}{x_{1}^{2}}\right| \\
& \leq 4 \pi \delta+\sup _{\left|x_{1}-x_{2}\right|<\delta} x_{2}^{2}\left|\sin \frac{1}{x_{1}^{2}}-\sin \frac{1}{x_{1}^{2}}\right| .
\end{aligned}
$$

Without loss of the generality, we may assume that $0<x_{2}<x_{1}$.

Case 8-1. $x_{2} \leq \delta^{\frac{1}{6}}$.

Then $x_{2}^{2}\left|\sin 1 / x_{1}^{2}-\sin 1 / x_{2}^{2}\right| \leq 2 \cdot \delta^{\frac{1}{3}}$.

Case 8-2. $x_{2}>\delta^{\frac{1}{6}}$.

Then there exists $\theta$ between $x_{1}$ and $x_{2}$ such that

$$
x_{2}^{2}\left|\sin \frac{1}{x_{1}^{2}}-\sin \frac{1}{x_{2}^{2}}\right|=x_{2}^{2}\left(x_{1}-x_{2}\right)\left|\cos \frac{1}{\theta^{2}}\right| \cdot \frac{1}{\theta^{3}} \leq 4 \pi^{2} \cdot \delta \cdot 1 \cdot \frac{1}{\delta^{\frac{1}{2}}}=2 \pi \delta^{\frac{1}{2}}
$$

holds.

Hence, by Case 8-1 and Case 8-2, we have the following inequality:

$$
\sup _{\left|x_{1}-x_{2}\right|<\delta} x_{2}^{2}\left|\sin \frac{1}{x_{1}^{2}}-\sin \frac{1}{x_{2}}\right| \leq 4 \pi^{2}\left(\delta^{\frac{1}{2}}+\delta^{\frac{1}{3}}\right) .
$$

So, by (1) and (2) we have

$$
\omega(\delta, s)|\log \delta| \leq\left(4 \pi \delta+4 \pi^{2}\left(\delta^{\frac{1}{2}}+\delta^{\frac{1}{3}}\right)\right)|\log \delta|,
$$

for any $\delta>0$. Therefore $\omega(\delta, s) \log \delta \rightarrow 0$ as $\delta \rightarrow 0$. By the Dini-Lipschitz test, Proposition 8 follows.

Denjoy's process of recovering a primitive from its derivative takes a countable ordinal number of iterations. But Denjoy has constructed examples of differentiable functions for which the process lasts arbitrarily many steps below the first uncountable ordinal. In his construction, he used the function $d$ and transfinite induction. 
By a variant of his method and the Dini-Lipschitz test, we are able to prove the following theorem:

Theorem 9. For any $\alpha<\omega_{1}$, there exists a differentiable function on $\mathbb{T}$ such that

$$
|f|_{Z}=1 \text { and }|f|_{D J}=|f|_{K W}=\alpha+1 .
$$

Proof of Theorem 9. Use induction on $\alpha$. We divide into three cases.

Case 9-1. $\alpha=0$.

Nothing to prove.

Case 9-2. $\alpha=1$.

Then the Denjoy rank of $d$ is 2. By Proposition $8,|d|_{Z}=1$.

Case 9-3. $\alpha \geq 2$.

We need the following lemma:

Lemma 10. Let $\alpha$ be a countable ordinal bigger than 1 . Then one can construct a function $f$ in $D(\mathbb{T})$ such that

(10-a) $f(0)=f^{\prime}(0)=0$ and $\|f\| \leq 1$;

(10-b) for any $0<\delta<1 / 1996$,

$$
\omega(\delta, f)|\log \delta| \leq \frac{1}{\log |\log \delta|}
$$

(10-c) for any $\delta>0, \partial_{f}^{D J}(\mathbb{T}, 1)=\partial_{f, \delta}^{K W}(\mathbb{T}, 1) \in \mathcal{K}_{\aleph_{0}}(\mathbb{T})$;

$(10-d)\left|\partial_{f}^{D J}(\mathbb{T}, 1)\right|_{C B}=\alpha+1$

(10-e) for any ordinal $\gamma, \partial_{f}^{D J}(\mathbb{T}, \gamma)=\partial^{C B}\left(\partial_{f}^{D J}(\mathbb{T}, 1), \gamma-1\right)$, where $\gamma-1=\gamma$ if $\gamma$ is a limit ordinal;

$(10-f)$ if $\partial_{f}^{D J}(\mathbb{T}, \gamma) \neq \emptyset$, then $0 \in \partial_{f}^{D J}(\mathbb{T}, \gamma)$.

This lemma goes through by transfinite induction. Roughly speaking, the basic constructional ideas of Lemma 10 are as follows:

(A) cut the unit circle into infinitely many intervals;

(B) glue an appropriate function on each interval obtained from (A) (we then get a function on the unit circle);

(C) (if it is necessary) add an extra function to the function obtained from (B).

At a limit stage, (A) and (B) are enough to build the function in Lemma 10. But at a successor stage we also need $(\mathrm{C})$, since the function from (A) and (B) does not satisfy the condition (10-e) in Lemma 10. We will then overcome this problem by the process $(\mathrm{C})$.

We will also use these ideas in the next section.

Proof of Lemma 10. Use transfinite induction on $\alpha$. As usual, we divide into three cases. For each $n \in \mathbb{N}$, set

$$
J_{n}=[1 /(n+1), 1 / n]
$$

Case 10-1. $\alpha=2$. 
We will need to use the processes (A), (B) and (C). We use the function $d$. We build an extra function for the process $(\mathrm{C})$. For this, we need to find a sequence $\left\langle h_{n}\right\rangle_{n \in \mathbb{N}}$ and $M>0$ such that for each $n \in \mathbb{N}(n \geq 1996)$,

(10-1-a) $h_{n}$ is differentiable on $J_{n}$;

(10-1-b) $h_{n}^{\prime}$ is continuous;

$(10-1-c) h_{n}^{\prime}(1 / n)=h_{n}^{\prime}(1 /(n+1))=0$;

$(10-1-\mathrm{d})$

$$
\begin{aligned}
h_{n}\left(\frac{1}{n}\right) & =\frac{(-1)^{n}}{n \log n \log \log n}, \\
h_{n}\left(\frac{1}{n+1}\right) & =\frac{(-1)^{n+1}}{(n+1) \log (n+1) \log \log (n+1)}, \\
\left\|h_{n}\right\| & =\frac{1}{n \log n \log \log n} ;
\end{aligned}
$$

(10-1-e) for all $x \in J_{n}$,

$$
\left|h_{n}^{\prime}(x)\right| \leq M \frac{n}{\log n \log \log n}
$$

for some fixed $M>1$.

Obviously, we can choose a sequence of functions $\left\langle h_{n}\right\rangle$ which satisfy the conditions (10-1-a), (10-1-b), (10-1-c), and (10-1-d). Also, by the condition (10-1-d),

$$
\begin{aligned}
\left|\frac{h_{n}\left(\frac{1}{n}\right)-h_{n}\left(\frac{1}{n+1}\right)}{\frac{1}{n}-\frac{1}{n+1}}\right| & =\frac{\frac{1}{n \log n \log \log n}+\frac{1}{(n+1) \log (n+1) \log \log (n+1)}}{\frac{1}{n}-\frac{1}{n+1}} \\
& =\frac{(n+1) \log (n+1) \log \log (n+1)+n \log n \log \log n}{\log n \log \log n \log (n+1) \log \log (n+1)} \\
& \leq \frac{2(n+1)}{\log n \log \log n} .
\end{aligned}
$$

Hence, we may find $\left\langle h_{n}\right\rangle$ and $M>0$ which fulfill all conditions as required. Define

$$
h(x)= \begin{cases}h_{n}(x), & x \in J_{n} \\ 0, & x=0 .\end{cases}
$$

Then clearly $h(x)$ is differentiable at $x \neq 0$. For the differentiability at $x=0$, we compute the following:

$$
\begin{aligned}
\left|\lim _{x \rightarrow 0} \frac{h(x)}{x}\right| & \leq \lim _{n \rightarrow \infty} \sup _{\frac{1}{n+1} \leq x \leq \frac{1}{n}} \frac{|h(x)|}{x} \leq \lim _{n \rightarrow \infty} \sup _{\frac{1}{n+1} \leq x \leq \frac{1}{n}} \frac{1}{x} \cdot \frac{1}{n \log n \log \log n} \\
& \leq \lim _{n \rightarrow \infty} \frac{n+1}{n \log n \log \log n}=0 .
\end{aligned}
$$

Therefore, $h$ is differentiable at $x=0$. So $h \in D(\mathbb{T})$. The function $h$ will play a role for the process (C). Now let $d_{n}$ be a scaled copy of the function $d$ which fits exactly in the closed interval $J_{n}$, i.e., for each $x \in J_{n}$,

$$
\left.d_{n}(x)=d(2 \pi n((n+1) x-1))\right) .
$$


Then define

$$
f(x)= \begin{cases}h(x)+d_{n}(x) /(n+1)^{2}, & \text { if } x \in J_{n} ; \\ 0, & \text { if } x=0 .\end{cases}
$$

Clearly, $f$ is differentiable on $D(\mathbb{T})$ and $f(0)=f^{\prime}(0)=0$. We obtain

$$
\{1 / n: n \geq 1\} \subseteq \partial_{f}^{D J}(\mathbb{T}, 1) \subseteq\{0\} \cup\{1 / n: n \geq 1\},
$$

since for any open set $U_{n}$ containing $1 / n$,

$$
\int_{U_{\frac{1}{n}} \cap J_{n}}\left|d_{n}^{\prime}(x)\right| d x=\infty \text { and } \int_{U_{\frac{1}{n}} \cap J_{n}}\left|h^{\prime}(x)\right| d x<\infty .
$$

So $\partial_{f}^{D J}(\mathbb{T}, 1)=\{0\} \cup\{1 / n: n \geq 1\}$. Since $\sum 1 /(n \log n \log \log n)=\infty, \partial_{f}^{D J}(\mathbb{T}, 2)=$ $\{0\}$ and so $\partial_{f}^{D J}(\mathbb{T}, 3)=\emptyset$. For any $\delta>0$, we easily get $\partial_{f, \delta}^{K W}(\mathbb{T}, 1)=$ the first $D J$ derived set of $\mathbb{T}$. So far, we get conditions (10-c), (10-d), (10-e), and (10-f) in Lemma 10. For the proof of Case 10-1, we will need two sublemmas.

Sublemma 11. For each positive number $\delta<1 / 1996$,

$$
\omega(\delta, h)|\log \delta|=O\left(\frac{1}{\log |\log \delta|}\right) .
$$

Proof of Sublemma 11. We fix a positive number $\delta<1 / 1996$. By the definition of the modulus of continuity, we have the following:

$$
\begin{aligned}
\omega(\delta, h) \leq & \sup _{\left|x_{1}-x_{2}\right|<\delta}\left|h\left(x_{1}\right)\right|+\left|h\left(x_{2}\right)\right|+\sup _{\substack{x_{1}<x_{2}<\delta<x_{2} \text { or } \\
x_{2}<\delta<x_{1} \\
\left|x_{1}-x_{2}\right|<\delta}}\left|h\left(x_{1}\right)-h\left(x_{2}\right)\right| \\
& +\sup _{\substack{\left|x_{1}-x_{2}\right|<\delta \\
x_{1}, x_{2}>\delta}}\left|h\left(x_{1}\right)-h\left(x_{2}\right)\right| .
\end{aligned}
$$

We take $n \in \mathbb{N}$ such that $1 /(n+1) \leq \delta<1 / n$. Let $m$ be a positive integer less than $n$. For fixed numbers $M_{0}, M_{1}$, and a number $\theta$ between $x_{1}$ and $x_{2}$, we then obtain

$$
\begin{aligned}
\left|h_{m}\left(x_{1}\right)-h_{m}\left(x_{2}\right)\right| & \leq\left|x_{1}-x_{2}\right| \cdot\left|h_{m}^{\prime}(\theta)\right| \leq\left|x_{1}-x_{2}\right| M_{0} \frac{m}{\log m \log \log m} \\
& \leq \frac{1}{n} M_{0} \sup _{m \leq n} \frac{m}{\log m \log \log m}<M_{1} \frac{1}{\log n \log \log n} .
\end{aligned}
$$

For the points $x_{1}$ and $x_{2}$ around $\delta$, we get

$$
\sup _{\substack{x_{1}<\delta<x_{2} \text { or } \\ x_{2}<\delta<x_{1} \\\left|x_{1}-x_{2}\right|<\delta}}\left|h\left(x_{1}\right)-h\left(x_{2}\right)\right| \leq M_{2} \frac{1}{\log n \log \log n}
$$

and

$$
\sup _{\substack{\left|x_{1}-x_{2}\right|<\delta \\ x_{1}, x_{2}<\delta}}\left|h\left(x_{1}\right)\right|+\left|h\left(x_{2}\right)\right| \leq M_{3} \frac{1}{n \log n \log \log n},
$$

for some fixed $M_{2}$ and $M_{3}$. By (3), (4), (5) and (6), we finally obtain

$$
\omega(\delta, h)|\log \delta|=O\left(\frac{1}{\log |\log \delta|}\right) .
$$

We have finished the proof of Sublemma 11. 
We now want to show that the function

$$
g(x)= \begin{cases}g_{n}(x) /(n+1)^{2}, & \text { if } x \in J_{n} ; \\ 0, & \text { otherwise }\end{cases}
$$

has the same property $(7)$ as $h$.

Sublemma 12. For each positive number $\delta<1 / 1996$,

$$
\omega(\delta, g)|\log \delta|=O\left(\frac{1}{\log |\log \delta|}\right) .
$$

Proof of Sublemma 12. We fix a positive number $\delta<1 / 1996$. Suppose $\left|x_{1}-x_{2}\right|<\delta$. Without loss of the generality, $x_{1}<x_{2}$.

Case 12-1. $x_{1}, x_{2} \in J_{n}$ for some $n$.

Then we have

$$
\begin{aligned}
\left|g\left(x_{2}\right)-g\left(x_{1}\right)\right| & \leq \sum_{i=1}^{n(n+1)}\left|g\left(x_{1}+\frac{i\left(x_{2}-x_{1}\right)}{n(n+1)}\right)-g\left(x_{1}+\frac{(i-1)\left(x_{2}-x_{1}\right)}{n(n+1)}\right)\right| \\
& \leq \sum_{i=1}^{n(n+1)} \frac{1}{(n+1)^{2}} \omega\left(g_{n}, \delta\right) \\
& \leq \omega\left(g_{n}, \delta\right) .
\end{aligned}
$$

By (10-b) and (8), for each $x_{1}, x_{2} \in J_{n}$ we obtain

$$
\left|g\left(x_{2}\right)-g\left(x_{1}\right)\right| \leq \frac{1}{|\log \delta| \log |\log \delta|}
$$

for each $x_{1}, x_{2} \in J_{n}$.

Case 12-2. $x_{1} \in J_{m}$ and $x_{2} \in J_{n}$ for some $m, n(m \neq n)$.

Say $n<m$. By the triangle inequality, we have

(10) $\left|g\left(x_{1}\right)-g\left(x_{2}\right)\right| \leq\left|g\left(x_{1}\right)-g\left(\frac{1}{m}\right)\right|+\left|g\left(\frac{1}{m}\right)-g\left(\frac{1}{n+1}\right)\right|+\left|g\left(\frac{1}{n+1}\right)-g\left(x_{2}\right)\right|$.

Note that $g(1 /(k+1))=0$ for all $k \in \mathbb{N}$. Hence, from (10) we have

$$
\begin{aligned}
\left|g\left(x_{1}\right)-g\left(x_{2}\right)\right| & \leq\left|g\left(x_{1}\right)-g\left(\frac{1}{m}\right)\right|+\left|g\left(\frac{1}{n+1}\right)-g\left(x_{2}\right)\right| \\
& \leq 2 \frac{1}{|\log \delta| \log |\log \delta|}
\end{aligned}
$$

since we follow the same method as in Case 12-1.

Case 12-3. $x_{1}=0$ and $x_{2} \in J_{n}$ for some $n$.

Then, clearly

$$
\left|g\left(x_{2}\right)-g\left(x_{1}\right)\right|=\left|g\left(x_{2}\right)\right| \leq 1 /(n+1)^{2} \leq \delta .
$$

From (9), (11), and (12), we finally have

$$
\omega(g, \delta)|\log \delta|=O\left(\frac{1}{\log |\log \delta|}\right) .
$$

We have finished the proof of Sublemma 12. 
By Sublemma 11 and Sublemma 12, we also have

$$
\omega(\delta, f)|\log \delta|=O\left(\frac{1}{\log |\log \delta|}\right) .
$$

The conditions in Lemma 10 (except for (10-a) and (10-b)) are not changed after we multiply $f$ by a positive number. Note that for any $a>0, \omega(\delta, a f)=a \omega(\delta, f)$. We take a small enough positive constant so that the conditions (10-a) and (10-b) hold. We have finished Case 10-1.

Case 10-2. $\alpha$ is a countable limit ordinal.

We will need the processes (A) and (B) only. We take a sequence of ordinals $\left\langle\beta_{n}\right\rangle_{n \in \mathbb{N}}$ such that for each $n \in \mathbb{N}, \beta_{n}<\beta_{n+1}$ and $\lim _{n \rightarrow \infty} \beta_{n}=\alpha$. By transfinite induction, we choose a sequence of functions $\left\langle f_{\beta_{n}}\right\rangle_{n \in \mathbb{N}}$ such that for each $n \in \mathbb{N}, f_{\beta_{n}}$ fulfills the conditions in Lemma 10. We define

$$
f(x)=\left\{\begin{array}{l}
\left.f_{\beta_{n}}(2 \pi n(n+1) x-1)\right) /(n+1)^{2}, \quad x \in J_{n} \\
0, \quad x=0 .
\end{array}\right.
$$

Then $f \in D(\mathbb{T})$. Clearly (10-a) holds. It is easy to see that for any $m \in \mathbb{N}$ and any $n \geq m$,

$$
\frac{1}{n+2} \in \partial_{f}^{D J}\left(\mathbb{T}, \beta_{m}\right) \text { and hence } 0 \in \partial_{f}^{D J}(\mathbb{T}, \alpha)
$$

But for any $n$,

$$
\partial_{f_{n}}^{D J}(\mathbb{T}, \alpha+1)=\emptyset \text { and so is } \partial_{f}^{D J}(\mathbb{T}, \alpha+1) .
$$

By the transfinite inductive assumption,

$$
\partial^{C B}\left(\partial_{f}^{D J}(\mathbb{T}, 1), \alpha+1\right)=\emptyset \text { but } \partial^{C B}\left(\left(\partial_{f}^{D J}(\mathbb{T}, 1), \alpha\right)\right)=\{0\} .
$$

Hence, we have shown (10-a), (10-c), (10-d), (10-e), and (10-f).

Sublemma 13. For each positive number $\delta<1 / 1996$,

$$
\omega(f, \delta)|\log \delta|=O\left(\frac{1}{\log |\log \delta|}\right) .
$$

Proof of Sublemma 13. The proof of Sublemma 13 is the same as the proof of Sublemma 12 .

Then by Sublemma 13, we complete Case 10-2 as in Case 10-1.

Case 10-3. $\alpha=\beta+1$ for some countable ordinal $\beta$.

We will need to use the processes (A), (B), and (C). The proof of this case follows the same methods as in Case 10-1 and Case 10-2.

By Cases 10-1, 10-2, and 10-3, we complete the proof of Lemma 10.

Now we complete the proof of Theorem 9. We fix an ordinal $\alpha \geq 2$. By Lemma 10, we then obtain a function $f$ fulfilling all conditions in Lemma 10. By the DiniLipschitz test, the condition (10-b) asserts the uniform convergence of the Fourier series of $f$. Namely, $|f|_{Z}=1$. From (10-d) and (10-e) in Lemma 10, the Denjoy rank of $f$ is $\alpha+1$. By Remark 7, the Kechris-Woodin rank of $f$ is at most $\alpha+1$. By Theorem $\mathrm{R}$, the Kechris-Woodin rank of $f$ should be $\alpha+1$. Therefore, we have finished the proof of Case 9-3.

By Cases 9-1, 9-2, and 9-3, we conclude the proof of Theorem 9. 


\section{Construction OF FUNCTIONS IN $D(\mathbb{T})$ HAVING ARBITRARILY BAD ZALCWASSER RANK WITH DENJOY RANK 2}

In the previous section, we have constructed for each countable ordinal $\alpha$ a differentiable function $f$ whose Denjoy rank (also Kechris-Woodin rank) is $\alpha+1$ but whose Zalcwasser rank is 1 . In the present section, we will demonstrate how to build for each countable ordinal $\alpha$ a differentiable function whose Zalcwasser rank (even Kechris-Woodin rank) is $\alpha+1$ but with Denjoy rank equal to 2 . Then, this proves that the Denjoy rank and the Zalcwasser rank are incomparable. We state our main theorem.

Theorem 14. Let $\alpha$ be countable ordinal. Then one can construct a function in $D(\mathbb{T})$ such that the Zalcwasser rank and the Kechris-Woodin rank of $f$ are $\alpha+1$ but the Denjoy rank of $f$ is 2 .

By the Boundedness Principle, our main theorem implies the following corollary:

Corollary 15. EC and $D(\mathbb{T})$ are $\Pi_{1}^{1}$ but non-Borel.

We need the following formula for Fourier series which appeared in [Zy]. For a measurable function $f$, we define

$$
\phi_{x}(f, t)=\frac{f(x+t)+f(x-t)-2 f(x)}{\pi}
$$

for all $x, t$.

Proposition 16. Let $\theta$ be a fixed positive number less than $\pi$. Then

$$
S_{n}(f, x)-f(x)=\int_{0}^{\theta} \phi_{x}(f, t) \frac{\sin n t}{t} d t+o(1) .
$$

On every interval where $f$ is bounded, the difference is bounded by a function of the norm of $f$, and converges uniformly to 0 with $n$.

Let $g$ be a measurable function, $P$ a subset in $\mathbb{T}$ and $x \in P$. For $\theta>0$, set

$$
\Omega(\theta, x, g, P)=\inf _{\delta>0} \inf _{p \in \mathbb{N}} \sup \left\{\left|\int_{0}^{\theta} \phi_{y}(g, t) \frac{\sin n t}{t} d t\right|: n \geq p,|x-y|<\delta \text { and } y \in P\right\} .
$$

Using Proposition 16, we get

Lemma 17. Let $\theta$ be a fixed positive number less than $\pi$. Suppose $g$ is in EC. Let $P$ be closed in $\mathbb{T}$ and $x \in P$. Then for any $t$ in $(0, \pi)$,

$$
\begin{aligned}
& \Omega(\theta, x, g, P)=\Omega(t, x, g, P) \text { and } \\
& \frac{1}{2} \omega(x, g, P) \leq \Omega(\theta, x, g, P) \leq \omega(x, g, P) .
\end{aligned}
$$

This lemma allows us to define a new oscillation function in $E C$ as follows: for $g \in E C, P$ closed in $\mathbb{T}$ and $x \in P$,

$$
\Omega(x, g, P)=\inf _{0<\theta<\pi} \Omega(\theta, x, g, P) .
$$


In fact, $\Omega(x, g, P)=\Omega(\theta, x, g, P)$ for all $0<\theta<\pi$. Let $a, b$ be real numbers. We define a polynomial $F_{a, b}$

$$
F_{a, b}(x)= \begin{cases}x^{2}(x-a)^{2}(x-b)^{2}, & x \in[a, b] \\ 0, & \text { otherwise }\end{cases}
$$

Then $F_{a, b}$ is differentiable everywhere. Let $P$ be a closed subset of $\mathbb{T}$ and $h$ be a function. We define $h_{a, b}$ and $P_{a, b}$ as follows:

$$
h_{a, b}(x)=h(a(x-b)) \text { and } P_{a, b}=\{b+x / a: x \in P\} .
$$

Lemma 18. Let $0<a<b<2 \pi$. Let $h$ be in EC and $P$ closed. Suppose $\omega(d, h, P)=\infty$. Let $c \in(a, b)$ and $N \in \mathbb{N}$. Then the oscillation function of $F_{a, b} h_{N, c}$ at $c+d / N$ is infinity, i.e., $\Omega\left(c+d / N, F_{a, b} h_{N, c}, P_{N, c}\right)=\infty$.

Recall the processes (A), (B), and (C). We will use these processes and transfinite induction to prove our main theorem.

For the same reason, the processes (A) and (B) will be enough at a limit stage in our proof of Theorem 14. We do not need any extra work at a limit stage.

In the previous section, we used the Dini-Lipschitz test to keep the Zalcwasser rank 1 in the proof of Theorem 9. We need to find a method like the Dini-Lipschitz test to make the Denjoy rank be only 2 while we are constructing arbitrarily bad Zalcwasser ranks. In our proof of Theorem 14, it will be required only at a successor stage.

At a successor stage, we will use the process (C) with (A) and (B). Namely, an extra function is required. The extra function will cause a problem in keeping the Denjoy rank equal to 2. However, taking advantage of the freedom of $N$ in Lemma 18 , we will develop a technical lemma which reduces this problem.

Proof of Lemma 18. By Lemma 17, it is enough to calculate

$$
\Omega\left(c+d / N, F_{a, b} h_{N, c}, P_{N, c}\right)=\infty .
$$

We fix $\theta$ in $(0, \pi)$. We obtain that for each $x, x \pm t \in[a, b]$,

$$
\begin{aligned}
\pi \phi_{x}\left(F_{a, b} h_{N, c}, t\right)= & F_{a, b}(x+t) h_{N, c}(x+t)+F_{a, b}(x-t) h_{N, c}(x-t) \\
& -2 F_{a, b}(x) h_{N, c}(x) \\
= & \left(h_{N, c}(x+t)+h_{N, c}(x-t)-2 h_{N, c}(x)\right) F_{a, b}(x) \\
& +\left(F_{a, b}(x+t)-F_{a, b}(x)\right) h_{N, c}(x+t) \\
& +\left(F_{a, b}(x-t)-F_{a, b}(x)\right) h_{N, c}(x-t) \\
= & \pi \phi_{x}\left(h_{N, c}, t\right) F_{a, b}(x) \\
& +\left(F_{a, b}(x+t)-F_{a, b}(x)\right) h_{N, c}(x+t) \\
& +\left(F_{a, b}(x-t)-F_{a, b}(x)\right) h_{N, c}(x-t)
\end{aligned}
$$

$\left(x_{t}\right.$ is in $(x, x+t), y_{t}$ in $\left.(x-t, x)\right)$

$$
\begin{aligned}
= & \pi \phi_{x}\left(h_{N, c}, t\right) F_{a, b}(x)+t F_{a, b}^{\prime}\left(x_{t}\right) h_{N, c}(x+t) \\
& +t F_{a, b}^{\prime}\left(y_{t}\right) h_{N, c}(x-t)
\end{aligned}
$$


(since $F_{a, b}^{\prime}, h_{N, c}$ are continuous)

$$
=\pi \phi_{x}\left(h_{N, c}, t\right) F_{a, b}(x)+t O(1) .
$$

Hence, from (13) we have

$$
\phi_{x}\left(F_{a, b} h_{N, c}, t\right)=\phi_{x}\left(h_{N, c}, t\right) F_{a, b}(x)+t O(1) .
$$

Fix $\delta>0, p \in \mathbb{N}$ and $N \in \mathbb{N}$. We calculate the following:

$$
\begin{array}{r}
\sup \left\{\left|\int_{0}^{\theta} \phi_{y}\left(F_{a, b} h_{N, c}, t\right) \frac{\sin m t}{t} d t\right|: m \geq p,|y-c-d / N|<\delta \text { and } y \in P\right\} \\
=\sup \left\{\left|\int_{0}^{\theta} F_{a, b}(y) \phi_{N(y-c)}(h, N t) \frac{\sin m t}{t} d t\right|: m \geq p,|N(y-c)-d|<N \delta\right. \\
\quad \text { and } N(y-c) \in P\}+O(\theta) \\
=\sup \left\{\left|\int_{0}^{\theta} F_{a, b}(c+z / N) \phi_{z}(h, N t) \frac{\sin m t}{t} d t\right|: m \geq p,|z-d|<N \delta\right. \\
\quad \geq \sup \left\{\left|\int_{0}^{\frac{\theta}{N}} F_{a, b}(c+z / N) \phi_{z}(h, t) \frac{\sin n t}{t} d t\right|: m \geq \frac{p}{N},|z-d|<N \delta\right. \\
\quad \text { and } z \in P\}+O(\theta)
\end{array}
$$

By the assumption, we have

$$
\sup \left\{\left|\int_{0}^{\frac{\theta}{N}} \phi_{z}(h, t) \frac{\sin n t}{t} d t\right|: n \geq \frac{p}{N},|z-d|<N d \text { and } z \in P\right\}=\infty .
$$

We let $M$ be a positive number. Hence we can find $n \geq p$ and $z$ close enough to zero so that

$$
\left|\int_{0}^{\frac{\theta}{N}} \phi_{z}(h, t) \frac{\sin n t}{t} d t\right|>M
$$

From (15) and (16), we obtain

$$
\left|\int_{0}^{\theta} \phi_{c+z / N}\left(F_{a, b} h_{N, c}, t\right) \frac{\sin n t}{t} d t\right| \geq F_{a, b}(c+z / N)(M+O(\theta)) .
$$

But $F_{a, b}(c+z / N) \geq C>0$ for all $z$ close enough to 0 where $C$ is a fixed constant. Note that $O(\theta)$ in (17) depends only on the norm of $h$. Since $M$ is arbitrary, from (15) and (17), we obtain

$$
\sup \left\{\left|\int_{0}^{\theta} \phi_{y}\left(F_{a, b} h_{N, c}, t\right) \frac{\sin m t}{t} d t\right|: m \geq p,|y-c-d / N|<\delta \text { and } y \in P\right\}=\infty .
$$

This completes the proof of Lemma 18.

The first step in proving Theorem 14 is to construct a differentiable function whose Fourier series converges uniformly except for a single point. Then by transfinite induction, we will finish all the constructions. 
For each $k \in \mathbb{N}$, set

$$
\alpha_{k}=\alpha^{-k}, \quad \frac{1}{2} N_{k}=n_{k}=4^{\alpha^{k} k}, \quad \alpha=2,3,4, \cdots .
$$

We introduce the Fejér sequence: for each $n<N$ in $\mathbb{N}$,

$$
R(x, N, n)=-\cos N x \sum_{i=1}^{n} \frac{\sin i x}{i} .
$$

Clearly

$$
\begin{aligned}
R(x, N, n)= & \frac{\sin (N-n) x}{n}+\frac{\sin (N-n+1) x}{n-1}+\cdots+\frac{\sin (N-1) x}{1} \\
& -\frac{\sin (N+1) x}{1}-\frac{\sin (N+2) x}{2}-\cdots-\frac{\sin (N+n) x}{n} .
\end{aligned}
$$

The Fejér polynomials $R(x, N, n)$ are bounded uniformly in $x, n, N$. We define a sequence of functions $\left\langle R_{\alpha, k}\right\rangle_{\alpha, k \in \mathbb{N}}$ as follows: for each $\alpha, k \in \mathbb{N}$,

$$
R_{\alpha, k}(x)=\alpha_{k} R\left(x, N_{k}, n_{k}\right) .
$$

$\left\|R_{\alpha, k}\right\|$ can be arbitrarily small (dependent on $\alpha$ ), since

$$
\left\|R_{\alpha, k}\right\| \leq \alpha_{k} \sup _{x}\left|R\left(x, N_{k}, n_{k}\right)\right| \leq \alpha^{-k} C
$$

for some fixed constant $C$. The $N_{k}$ th partial Fourier sum of $R_{\alpha, k}(x)$ is

$$
\alpha_{k}\left(\frac{\sin \left(N_{k}-n_{k}\right) x}{n_{k}}+\cdots+\frac{\sin \left(N_{k}-1\right) x}{1}\right) .
$$

Therefore, for $x_{k}=\pi / 4 n_{k}$ we get

$$
\alpha_{k}\left(\frac{\sin \left(N_{k}-n_{k}\right) x_{k}}{n_{k}}+\cdots+\frac{\sin \left(N_{k}-1\right) x_{k}}{1}\right) \geq \alpha_{k} \frac{1}{\sqrt{2}} \sum_{i=1}^{n_{k}} \frac{1}{i} \geq k \frac{\log 2}{\sqrt{2}} .
$$

Set

$$
a_{k}=\frac{1}{2} x_{k} \text { and } b_{k}=2 x_{k} .
$$

It is easy to see that for each $k \in \mathbb{N}$,

$$
b_{k+1}<a_{k}<x_{k}<b_{k} .
$$

We take $\left\{m_{k}\right\}_{k \in \mathbb{N}}$ such that for each $k \in \mathbb{N}$,

$$
x_{k}+x_{m_{k}} \in\left(a_{k}, b_{k}\right), \quad m_{k}<m_{k+1} \text { in } \mathbb{N}
$$

and

$$
\begin{gathered}
\alpha_{m_{k}}\left(\frac{\sin \left(N_{m_{k}}-n_{m_{k}}\right) x_{m_{k}}}{n_{m_{k}}}+\cdots+\frac{\sin \left(N_{m_{k}}-1\right) x_{m_{k}}}{1}\right) \\
\quad \geq \alpha_{m_{k}} \frac{1}{\sqrt{2}} \sum_{i=1}^{n_{m_{k}}} \frac{1}{i} \\
\quad \geq \frac{k}{\left(x_{k}+x_{m_{k}}\right)^{2}\left(x_{k}+x_{m_{k}}-a_{k}\right)^{2}\left(x_{k}+x_{m_{k}}-b_{k}\right)^{2}} .
\end{gathered}
$$

Let $s$ be an increasing function from $\mathbb{N}$ to $\mathbb{N}$. For each $k \in \mathbb{N}$, we set

$$
B_{k}(x)=R_{s(k), m_{k}}\left(x-x_{k}\right), c_{k}=x_{k}+x_{m_{k}} \text { and } F_{k}(x)=F_{a_{k}, b_{k}}(x) .
$$


From (18), we then have

$$
\begin{gathered}
\alpha_{m_{k}}\left(\frac{\sin \left(N_{m_{k}}-n_{m_{k}}\right) x_{m_{k}}}{n_{m_{k}}}+\cdots+\frac{\sin \left(N_{m_{k}}-1\right) x_{m_{k}}}{1}\right) \\
\geq \frac{k}{c_{k}^{2}\left(c_{k}-a_{k}\right)^{2}\left(c_{k}-b_{k}\right)^{2}}=\frac{k}{F_{k}\left(c_{k}\right)} .
\end{gathered}
$$

Finally, we define a function $f_{s}$ on $\mathbb{T}$ as follows:

$$
f_{s}(x)= \begin{cases}F_{k}(x) B_{k}(x), & \text { if } x \in\left[a_{k}, b_{k}\right] \\ 0, & \text { otherwise. }\end{cases}
$$

Using some basic properties of the Fejér polynomial, we have the following proposition.

Proposition 19. Let $\varepsilon>0$. There exists an increasing sequence $s$ from $\mathbb{N}$ to $\mathbb{N}$ such that

$$
\begin{aligned}
& \text { (19-a) } f_{s}(0)=f_{s}^{\prime}(0)=0 ; \\
& (19-b)\left\|f_{s}\right\| \leq \varepsilon ; \\
& (19-c) \Omega\left(x, f_{s}, \mathbb{T}\right)=0 \text { for all } x \neq 0 \text { and } \Omega\left(0, f_{s},\{0\} \cup\left\{c_{k}\right\}_{k \in \mathbb{N}}\right)=\infty ; \\
& (19-d)\left|f_{s}\right|_{D J}=2 \text { and } \partial_{f_{s}}^{D J}(\mathbb{T}, 1)=\{0\} .
\end{aligned}
$$

By a non-constructive method, Ajtai and Kechris [AK] have shown that for any $\alpha<\omega_{1}$, there exists $f \in D(\mathbb{T})$ such that $|f|_{Z}>\alpha$. Yet, we do not know of any constructive example in the literature of a differentiable function on $\mathbb{T}$ whose Fourier series does not converge uniformly, not even a differentiable function whose Zalcwasser rank is 2 !

Our function in Proposition 19 will have two roles in our proof of Theorem 14. Its first role is as a differentiable function whose Zalcwasser rank and Denjoy rank are 2. The second role is as an extra function in the process $(\mathrm{C})$. Because of the way we construct $f_{s}$, we use Lemma 18 to develop a technical lemma. This technical lemma keeps the Denjoy rank equal to 2 while we carry out the process $(\mathrm{C})$.

Proof of Proposition 19. Let $\varepsilon>0$. We choose an increasing function $s$ from $\mathbb{N}$ to $\mathbb{N}$ such that for each $k \in \mathbb{N}, s(k)<\varepsilon \cdot \min \left(a_{k-1}-b_{k}, a_{k}-a_{k+1}\right)$. It is easy to see that $f_{s}$ is differentiable except at $x=0$. Since $\left\|B_{k}\right\|$ is bounded uniformly in $k$ and

$$
\frac{f_{s}(x)-f_{s}(0)}{x}= \begin{cases}B_{k}(x) F_{k}(x), & \text { if } x \in\left[a_{k}, b_{k}\right] ; \\ 0, & \text { otherwise },\end{cases}
$$

we obtain the following:

$$
\lim _{x \rightarrow 0}\left|\frac{f_{s}(x)-f_{s}(0)}{x}\right| \leq \lim _{k \rightarrow \infty}\left\|B_{k}\right\| \sup _{x \in\left[a_{k}, b_{k}\right]} \frac{F_{k}(x)}{x}=0 .
$$

So $f_{s}$ is differentiable on $\mathbb{T}$. By the construction of $f_{s}$, we immediately have that $\Omega\left(x, f_{s}, \mathbb{T}\right)=0$ for all $x \neq 0,\left\|f_{s}\right\| \leq \varepsilon, f_{s}(0)=f_{s}^{\prime}(0)=0$ and $\partial_{f_{s}}^{D J}(\mathbb{T}, 1) \subseteq\{0\}$. Suppose the condition $\Omega\left(0, f_{s},\{0\} \cup\left\{c_{k}\right\}_{k \in \mathbb{N}}\right)=\infty$ holds. Then $\partial_{f_{s}}^{D J}(\mathbb{T}, 1)$ should be nonempty (i.e., $\partial_{f_{s}}^{D J}(\mathbb{T}, 1)=\{0\}$ ) since $\partial_{f_{s}}^{D J}(\mathbb{T}, 1)=\emptyset$ implies that the function $f_{s}^{\prime}$ is integrable, i.e., the Fourier series of $f_{s}$ converges uniformly. Hence it suffices to show that $\Omega\left(0, f_{s},\{0\} \cup\left\{c_{k}\right\}_{k \in \mathbb{N}}\right)=\infty$. Let $\delta>0$ and $p \in \mathbb{N}$. Choose $N_{0} \in \mathbb{N}$ 
such that $x_{k}<\delta$ for all $k \geq N_{0}$ and $N_{0}>p$. Then we obtain

$$
\begin{aligned}
\left|\int_{0}^{\theta} \phi_{c_{k}}\left(f_{s}, t\right) \frac{\sin n t}{t} d t\right| & \geq\left|\int_{0}^{\theta} \phi_{c_{k}}\left(B_{k} F_{k}, t\right) \frac{\sin n t}{t} d t\right| \\
& -\left|\int_{0}^{\theta} \phi_{c_{k}}\left(f_{s}-B_{k} F_{k}, t\right) \frac{\sin n t}{t} d t\right| \\
& =A-B .
\end{aligned}
$$

It is easy to see that

$$
\begin{aligned}
B \leq\left(\frac{1}{\pi}\left|\int_{-c_{k}+a_{k-1}}^{-c_{k}+b_{k-1}} B_{k-1}\left(c_{k}+t\right) F_{k-1}\left(c_{k}+t\right) \frac{\sin n t}{t} d t\right|\right. \\
\left.+\frac{1}{\pi}\left|\int_{-c_{k}+a_{k-2}}^{-c_{k}+b_{k-2}} B_{k-2}\left(c_{k}+t\right) F_{k-2}\left(c_{k}+t\right) \frac{\sin n t}{t} d t\right|+\cdots\right) \\
+\left(\frac{1}{\pi}\left|\int_{c_{k}-a_{k+1}}^{c_{k}-b_{k+1}} B_{k+1}\left(c_{k}-t\right) F_{k+1}\left(c_{k}-t\right) \frac{\sin n t}{t} d t\right|\right. \\
\left.+\frac{1}{\pi}\left|\int_{c_{k}-a_{k+2}}^{c_{k}-b_{k+2}} B_{k+2}\left(c_{k}-t\right) F_{k+2}\left(c_{k}-t\right) \frac{\sin n t}{t} d t\right|+\cdots\right) .
\end{aligned}
$$

From (21), we then have

$$
\begin{aligned}
B \leq & L\left\{\left(\frac{b_{k-1}-a_{k-1}}{a_{k-1}-c_{k}} \cdot \frac{1}{s(k-1)^{k-1}}+\frac{b_{k-2}-a_{k-2}}{a_{k-2}-c_{k}} \cdot \frac{1}{s(k-2)^{k-2}}+\cdots\right)\right. \\
& \left.+\left(\frac{b_{k+1}-a_{k+1}}{c_{k}-a_{k+1}} \frac{1}{s(k+1)^{k+1}}+\frac{b_{k+2}-a_{k+2}}{c_{k}-a_{k+2}} \frac{1}{s(k+2)^{k+2}}+\cdots\right)\right\} \\
\leq & L\left\{\left(\frac{b_{k-1}-a_{k-1}}{s(k-1)^{k-2}}+\frac{b_{k-2}-a_{k-2}}{s(k-2)^{k-2}}+\cdots\right)\right. \\
& \left.+\left(\frac{b_{k+1}-a_{k+1}}{s(k+1)^{k}}+\frac{b_{k+2}-a_{k+2}}{s(k+2)^{k+1}}+\cdots\right)\right\} \leq L_{1},
\end{aligned}
$$

where $L$ and $L_{1}$ are fixed constants. From (20) and (22), we get

$$
\left|\int_{0}^{\theta} \phi_{c_{k}}\left(f_{s}, t\right) \frac{\sin n t}{t} d t\right| \geq\left|\int_{0}^{\theta} \phi_{c_{k}}\left(B_{k} F_{k}, t\right) \frac{\sin n t}{t} d t\right|-L_{1} .
$$

Note that the $N_{m_{k}}$ th partial Fourier sum of $B_{k}$ at $x_{k}+x_{m_{k}}$ is

$$
\int_{0}^{\theta} \phi_{x_{m_{k}}}\left(R_{s(k), m_{k}}, t\right) \frac{\sin N_{m_{k}} t}{t} d t+o(1) .
$$

By Proposition 16, $o(1)$ is uniformly bounded on $k$ and $x$, since the $B_{k}$ are uniformly bounded. From (19) and (24),

$$
\begin{aligned}
\left|\int_{0}^{\theta} \phi_{c_{k}}\left(B_{k}, t\right) \frac{\sin N_{m_{k}} t}{t} d t\right| & =\left|\int_{0}^{\theta} \phi_{x_{m_{k}}}\left(R_{s(n), m_{k}}, t\right) \frac{\sin N_{m_{k}} t}{t} d t\right|+o(1) \\
& \geq \frac{k}{F_{k}\left(c_{k}\right)}+o(1) .
\end{aligned}
$$


From (25), we obtain

$$
\left|\int_{0}^{\theta} \phi_{c_{k}}\left(B_{k} F_{k}, t\right) \frac{\sin N_{m_{k}} t}{t} d t\right| \geq k+O(1),
$$

since

$$
\phi_{c_{k}}\left(B_{k} F_{k}, t\right)-\phi_{c_{k}}\left(B_{k}, t\right) F_{k}\left(c_{k}\right)=t O(1) .
$$

It is not hard to see that $O(1)$ in (26) is uniformly bounded. From (23) and (26), we finally have

$$
\left|\int_{0}^{\theta} \phi_{c_{k}}\left(f_{s}, t\right) \frac{\sin n t}{t} d t\right| \geq k+O(1) .
$$

Therefore, we demonstrate that $\Omega\left(\theta, 0,\{0\} \cup\left\{c_{k}\right\}_{k \in \mathbb{N}}\right)=\infty$. This completes the proof of Proposition 19.

We need another technical lemma with Proposition 19 to do the process (C). It will follow from Lemma 18 and Proposition 19.

Let $\left\langle M_{n}\right\rangle$ be a sequence of positive integers such that

$$
\frac{2 \pi}{M_{k+1}}<\max \left\{c_{k}-c_{k+1}, c_{k+1}-c_{k+2}\right\}
$$

We define the transfinite sequence $\left\langle K_{\left\langle M_{n}\right\rangle}^{(\alpha)}\right\rangle_{\alpha<\omega_{1}}$. By transfinite induction, we let

$$
\begin{gathered}
K_{\left\langle M_{n}\right\rangle}^{(0)}=\{0\} \cup\left\{c_{k}\right\}_{k \in \mathbb{N}} ; \\
K_{\left\langle M_{n}\right\rangle}^{(\alpha)}=\{0\} \cup\left\{c_{k}\right\}_{k \in \mathbb{N}} \cup\left\{c_{k}+x / M_{k}: k \in \mathbb{N} \text { and } x \in K_{\left\langle M_{n}\right\rangle}^{(\beta)} \text { for some } \beta<\alpha\right\} .
\end{gathered}
$$

Note that for each countable ordinal $\alpha, K_{\left\langle M_{n}\right\rangle}^{(\alpha)}$ is countable. We will need the following lemma to overcome some problems while we do the process $(\mathrm{C})$. This lemma will play the same role as the Dini-Lipschitz test. Namely, it will keep Denjoy ranks 2 while we construct arbitrarily high Zalcwasser ranks.

Lemma 20. Let $\alpha$ be a countable ordinal. Let $\varepsilon>0$. Then there exist an increasing sequence of positive integers such that

(20-a) $f_{s}$ fulfills all conditions in Proposition 19;

(20-b) if $p$ is in $\mathcal{K}(\mathbb{T})$ with $P \subseteq K_{\left\langle M_{n}\right\rangle}^{(\alpha)}$ and $\left\langle\left(s_{n}, t_{n}\right)\right\rangle$ is the sequence of open intervals complementing $P$ in $\mathbb{T}$, then

$$
\sum_{P}\left|f_{s}\left(t_{n}\right)-f_{s}\left(s_{n}\right)\right| \leq \varepsilon
$$

where $\sum_{P}$ indicates that the sum is to be taken over all intervals $\left(s_{n}, t_{n}\right)$ of $\mathbb{T}$.

Proof of Lemma 20. We fix $\varepsilon>0$. By Proposition 19, we then have the function $f_{s}$ satisfying all conditions in Proposition 19. In addition, it is not hard to see that we may take $s$ in such a way that for each $k$,

$$
\left\|B_{k}\right\|\left\|F_{k}^{\prime}\right\|<\frac{\varepsilon}{2^{k+3}}
$$


Now we choose an increasing sequence of positive integers $\left\langle M_{n}\right\rangle$ (satisfying (27)) such that for each $k, M_{k}<M_{k+1}$ and $M_{k}$ is so large that for all $x, y \in \mathbb{T}$,

$$
\begin{aligned}
\left|B_{k}\left(c_{k}+\frac{x}{M_{k}}\right)-B_{k}\left(c_{k}+\frac{y}{M_{k}}\right)\right| & \leq \frac{|x-y|}{s(k)^{k} M_{k}}\left|R^{\prime}\left(\theta_{k, x, y}, N_{m_{k}}, n_{m_{k}}\right)\right| \\
& \leq \frac{\varepsilon|x-y|}{2^{k+3} 1996},
\end{aligned}
$$

where $\theta_{k, x, y}$ is between $x / M_{k}$ and $y / M_{k}$. It is possible since for each $k$ the function $R^{\prime}\left(x, N_{m_{k}}, n_{m_{k}}\right)$ is bounded. If $\alpha=0$, Lemma 20 follows immediately. Suppose $\alpha \geq 1$. Let $P$ be a closed subset of $K_{\left\langle M_{n}\right\rangle}^{(\alpha)}$. Let $\left\langle\left(s_{n}, t_{n}\right)\right\rangle$ be the sequence of open intervals complementing $P$ in $\mathbb{T}$. Note that

$$
\bigcup_{k \in \mathbb{N}}\left[a_{k}, b_{k}\right] \cap P \subseteq P \subseteq\{0\} \cup \bigcup_{k \in \mathbb{N}}\left[a_{k}, b_{k}\right] \cap P .
$$

By (30), it is enough to show that

$$
\sum_{\left[a_{k}, b_{k}\right] \cap P}\left|f_{s}\left(t_{n}\right)-f_{s}\left(s_{n}\right)\right| \leq \frac{\varepsilon}{2^{k+3}},
$$

where $\sum_{\left[a_{k}, b_{k}\right] \cap P}$ indicates that the sum is to be taken over all intervals $\left(s_{n}, t_{n}\right)$ which are contained in $\left[a_{k}, b_{k}\right]$. Note that

$$
\left[a_{k}, b_{k}\right] \cap P \subseteq\left\{c_{k}\right\} \cup\left\{c_{k}+\frac{x}{M_{k}}: x \in \mathbb{T}\right\} .
$$

From (31), we define

$$
P_{k}=\left\{x \in \mathbb{T}: c_{k}+\frac{x}{M_{k}} \in\left[a_{k}, b_{k}\right] \cap P\right\}
$$

for each $k \in \mathbb{N}$. Clearly, $P_{k}$ is a closed subset in $\mathbb{T}$. We let $\left\langle\left(s_{n}^{\prime}, t_{n}^{\prime}\right)\right\rangle$ be the sequence of open intervals complementing $P_{k}$ in $\mathbb{T}$. By definition of $f_{s}$, we obtain

$$
\begin{aligned}
\sum_{\left[a_{k}, b_{k}\right] \cap P}\left|f_{s}\left(t_{n}\right)-f_{s}\left(s_{n}\right)\right|= & \sum_{\left[a_{k}, b_{k}\right] \cap P}\left|F_{k}\left(t_{n}\right) B_{k}\left(t_{n}\right)-F_{k}\left(s_{n}\right) B_{k}\left(s_{n}\right)\right| \\
\leq & \sum_{\left[a_{k}, b_{k}\right] \cap P}\left|F_{k}\left(t_{n}\right)-F_{k}\left(s_{n}\right)\right|\left|B_{k}\left(t_{n}\right)\right| \\
& +\sum_{\left[a_{k}, b_{k}\right] \cap P}\left|F_{k}\left(s_{n}\right)\right|\left|B_{k}\left(t_{n}\right)-B_{k}\left(s_{n}\right)\right| \\
\leq & \sum_{\left[a_{k}, b_{k}\right] \cap P}\left\|B_{k}\right\|\left\|F_{k}^{\prime}\right\|\left|t_{n}-s_{n}\right| \\
& +\sum_{\left[a_{k}, b_{k}\right] \cap P}\left\|F_{k}\right\|\left|B_{k}\left(t_{n}\right)-B_{k}\left(s_{n}\right)\right| .
\end{aligned}
$$

From (28), (29), (31), (32) and (33), we have

$$
\begin{aligned}
& \sum_{\left[a_{k}, b_{k}\right] \cap P}\left|f_{s}\left(t_{n}\right)-f_{s}\left(s_{n}\right)\right| \\
& \quad \leq \frac{\varepsilon}{2^{k+3} 1996} \sum_{\left[a_{k}, b_{k}\right] \cap P}\left|t_{n}-s_{n}\right|+\frac{\varepsilon}{2^{k+3} 1996} \sum_{P_{k}}\left|t_{n}^{\prime}-s_{n}^{\prime}\right|,
\end{aligned}
$$


where $\sum_{P_{k}}$ indicates that the sum is to be taken over all intervals $\left(s_{n}^{\prime}, t_{n}^{\prime}\right)$ which are contained in $\mathbb{T}$. From (34), we finally obtain

$$
\begin{aligned}
\sum_{\left[a_{k}, b_{k}\right] \cap P}\left|f_{s}\left(t_{n}\right)-f_{s}\left(s_{n}\right)\right| & \leq \frac{\varepsilon}{2^{k+3} 1996}\left(b_{k}-a_{k}\right)+\frac{\varepsilon}{2^{k+3} 1996} 2 \pi \\
& \leq \frac{\varepsilon}{2^{k+1}} .
\end{aligned}
$$

Hence, we complete the proof of Lemma 20.

We are ready to prove our main theorem.

Proof of Theorem 14. We divide into three cases.

Case 14-1. $\alpha=1$.

By Proposition 8, it follows.

Case 14-2. $\alpha=2$.

By Proposition 19, it follows.

Case 14-3. $\alpha \geq 3$.

We claim the following lemma holds:

Lemma 21. Let $\alpha$ be a countable ordinal bigger than or equal to 1. Let $\varepsilon>0$. Then there exists a function $f$ in $D(\mathbb{T})$ such that

(21-a) $\|f\| \leq \varepsilon$;

(21-b) for any $\delta>0, \partial_{f, \delta}^{Z}(\mathbb{T}, 1)=\partial_{f, \delta}^{K W}(\mathbb{T}, 1) \in K_{\aleph_{0}}(\mathbb{T})$;

(21-c)

$$
\left|\partial_{f, \delta}^{Z}(\mathbb{T}, 1)\right|_{C B}= \begin{cases}\alpha+1, & \text { if } \alpha \text { is finite } \\ \alpha+2, & \text { otherwise }\end{cases}
$$

(21-d) $\Omega\left(0, f,\{0\} \cup\left\{c_{k}\right\}_{k \in \mathbb{N}}\right)=\infty$;

(21-e) for any $\delta>0, \partial_{f, \delta}^{Z}(\mathbb{T}, \alpha)=\{0\} \cup\left\{c_{k}\right\}_{k \in \mathbb{N}}$;

(21-f) for all $\beta$ and $\delta, \delta^{\prime}>0, \partial_{f, \delta}^{Z}(\mathbb{T}, \beta)=\partial_{f, \delta^{\prime}}^{Z}(\mathbb{T}, \beta)$;

(21-g) $|f|_{D J}=2$ and $\partial_{f}^{D J}(\mathbb{T}, 1)=\partial_{f}^{Z}(\mathbb{T}, 1)$;

$(21-h)$ if $\left\langle\left(a_{n}, b_{n}\right)\right\rangle$ is the sequence of open intervals complementing $\partial_{f}^{D J}(\mathbb{T}, 1)$ in $\mathbb{T}$, then

$$
\sum\left|f\left(t_{n}\right)-f\left(s_{n}\right)\right| \leq \varepsilon
$$

where $\sum$ indicates that the sum is to be taken over all intervals $\left(a_{n}, b_{n}\right)$ which are contained in $\mathbb{T}$.

Lemma 21 covers all successor stages of Case 14-3. So the processes (A), (B), and $(\mathrm{C})$ will be needed to prove Lemma 21. By using the processes (A) and (B), Lemma 21 also proves all limit stages of Case 14-3.

Proof of Lemma 21. We divide into three cases: We choose an increasing sequence of positive integers $\left\{M_{k}\right\}_{k \in \mathbb{N}}$ satisfying Lemma 20 for $\varepsilon / 2$. We say $H$ is the function obtained by Lemma 20 .

Case 21-1. $\alpha=1$. 
We will need to follow the processes (A), (B), and (C). Let $\varepsilon>0$. We choose $\left\{\varepsilon_{k}\right\}_{k \in \mathbb{N}}$ such that, $\varepsilon_{k}<\varepsilon / 2^{k+3}$. Then by Proposition 19 , there exists $\left\{f_{k}\right\}$ in $D(\mathbb{T})$ such that for all $k \in \mathbb{N}$,

(21-1-a) $f_{k}(0)=f_{k}^{\prime}(0)=0$;

(21-1-b) $\left\|f_{k}\right\| \leq \varepsilon_{k}$

(21-1-c) $\Omega\left(x, f_{k}, \mathbb{T}\right)=0$ for all $x \neq 0$ and $\Omega\left(0, f_{k},\{0\} \cup\left\{c_{k}\right\}_{k \in \mathbb{N}}\right)$;

(21-1-d) $\left|f_{k}\right|_{D J}=2$ and $\partial_{f_{k}}^{D J}(\mathbb{T}, 1)=\{0\}$.

We define a function $f_{*}$ on $D(\mathbb{T})$ as follows:

$$
f_{*}(x)= \begin{cases}F_{k}(x) f_{k}\left(M_{k}\left(x-c_{k}\right)\right), & \text { if } x \in\left[a_{k}, b_{k}\right] ; \\ 0, & \text { otherwise }\end{cases}
$$

As usual, $f_{*}$ is differentiable. It is easy to see that

$$
\partial_{f_{*}}^{D J}(\mathbb{T}, 1) \subseteq K_{\left\langle M_{n}\right\rangle}^{(0)}=\{0\} \cup\left\{c_{k}\right\}_{k \in \mathbb{N}} .
$$

By Lemma 18, we note that for each $k, \Omega\left(c_{k}, f_{*}, \partial_{f_{*}, \delta}^{Z}(\mathbb{T}, 1)\right)=\infty$ for all $\delta>0$. It is not hard to see that $f_{*}$ satisfies the conditions (21-a), (21-b), (21-c) and (21-e) in Lemma 21. In fact, it suffices to show (21-d) and (21-h). Obviously, $f_{*}\left(c_{k}\right)=0$ for all $k \in \mathbb{N}$. So $\left(21\right.$-h) holds for $f_{*}$. If $f_{*}$ already satisfies condition (21-d), we are done. Otherwise $\Omega\left(0, f_{*},\{0\} \cup\left\{c_{k}\right\}_{k \in \mathbb{N}}\right)<\infty$. Then we define a new function $f$ on $D(\mathbb{T})$ as follows:

$$
f(x)=f_{*}(x)+H(x) .
$$

Then by the constructions of $f_{*}$ and $H$, one can observe that $f$ fulfills (21-a), (21b), (21-c) and (21-e). By Lemma 20, (20-g) holds for $f$. Hence, it suffices to show (21-d). Let $\delta>0$ and $p \in \mathbb{N}$. We fix $\theta$ in $(0,1 / 1996)$. We note that

$$
\omega\left(0, H,\{0\} \cup\left\{c_{k}\right\}_{k \in \mathbb{N}}\right)=\infty .
$$

Then it is enough to establish the following:

Sublemma 22.

$$
\sup \left\{\left|\int_{0}^{\theta} \phi_{c_{k}}\left(f_{*}, t\right) \frac{\sin n t}{t} d t\right|: c_{k} \leq \delta \text { and } n \geq P\right\}<\infty .
$$

Proof of Sublemma 22. Suppose the supremum is infinite. Note that for any $\delta^{\prime}, p^{\prime}$,

$$
\sup \left\{\left|\int_{0}^{\theta} \phi_{c_{k}}\left(f_{*}, t\right) \frac{\sin n t}{t} d t\right|: \delta^{\prime} \leq c_{k} \leq \delta \text { and } p^{\prime} \geq n \geq p\right\}<\infty .
$$

Hence for arbitrarily large $M>0$, we can find arbitrarily small $c_{k}$ and arbitrarily large $n$ such that

$$
\left|\int_{0}^{\theta} \phi_{c_{k}}\left(f_{*}, t\right) \frac{\sin n t}{t} d t\right|>M .
$$

This immediately implies $\Omega\left(0, f_{*},\{0\} \cup\left\{c_{k}\right\}_{k \in \mathbb{N}}\right)=\infty$. This contradicts our assumption.

This completes the proof of Case 21-1.

Case 21-2. $\alpha$ is a successor ordinal, i.e., $\alpha=\beta+1$ for some $\beta<\omega_{1}$. 
By transfinite induction, we have a function $f_{\beta}$ which satisfies all conditions in Lemma 21. We define a function $f_{*}$ in $D(\mathbb{T})$ as follows:

$$
f_{*}(x)= \begin{cases}1 / 2^{k+3} F_{k}(x) f_{\beta}\left(M_{k}\left(x-c_{k}\right)\right), & \text { if } x \in\left[a_{k}, b_{k}\right] ; \\ 0, & \text { otherwise. }\end{cases}
$$

We note that

$$
\partial_{f_{*}}^{D J}(\mathbb{T}, 1) \subseteq K_{\left\langle M_{n}\right\rangle}^{(\alpha+1)} .
$$

By Lemma 20 and (35), we easily get (21-h) for $\varepsilon / 2$ and $f_{*}$. For $\alpha$ all conditions in Lemma 21 hold except for (21-d) and (21-f). So it suffices to show (21-d). Suppose (21-d) does not hold. As in Case 21-1, we then get a new function $f=f_{*}+H$ from $f_{*}$ and $H$ so that all conditions in Lemma 21 hold for $\alpha$. This completes the proof of Case 21-2.

Case 21-3. $\alpha$ is a limit ordinal.

Then we take a sequence of ordinals $\left\{\beta_{n}\right\}_{n \in \mathbb{N}}$ such that for each $n \in \mathbb{N}, \beta_{n}<\beta_{n+1}$ and $\lim _{n \rightarrow \infty} \beta_{n}=\alpha$. We fix a sequence of positive numbers $\left\langle\varepsilon_{n}\right\rangle$ such that for each $n, \varepsilon_{n}<\varepsilon / 2^{n+3}$. By transfinite induction, we take a sequence of functions $\left\{f_{\beta_{n}}\right\}$ in $D(\mathbb{T})$ such that for each $n, f_{\beta_{n}}$ satisfies all conditions in Lemma 21 for $\varepsilon_{n}$. We define a function $f_{1}$ on $D(\mathbb{T})$ as follows:

$$
f_{1}(x)= \begin{cases}F_{k}(x) f_{\beta_{n}}\left(M_{k}\left(x-c_{k}\right)\right), & \text { if } x \in\left[a_{k}, b_{k}\right] ; \\ 0, & \text { otherwise. }\end{cases}
$$

Clearly, $f_{1} \in D(\mathbb{T})$ and we have

$$
\partial_{f_{1}}^{Z}(\mathbb{T}, 1) \subseteq K_{\left\langle M_{n}\right\rangle}^{(\alpha+1)} .
$$

It is easy to show that for any $\delta>0$,

$$
0 \in \partial_{f_{1}, \delta}^{Z}(\mathbb{T}, \alpha) .
$$

We then define $f_{*}$ as follows:

$$
f_{*}(x)= \begin{cases}1 / 2^{k+3} F_{k}(x) f_{1}\left(M_{k}\left(x-c_{k}\right)\right), & \text { if } x \in\left[a_{k}, b_{k}\right] ; \\ 0, & \text { otherwise. }\end{cases}
$$

From (36), we have

$$
\partial_{f_{*}}^{Z}(\mathbb{T}, 1) \subseteq K_{\left\langle M_{n}\right\rangle}^{(\alpha+2)} .
$$

By the construction of $f_{*}$ and (37), $f_{*}$ satisfies (21-a), (21-b), (21-c), (21-e), (21-g), and (21-h) for $\varepsilon / 2$. Also, for all $\beta \leq \alpha$ and $\delta, \delta^{\prime}>0$

$$
\partial_{f_{*}, \delta}^{Z}(\mathbb{T}, \beta)=\partial_{f_{*}, \delta^{\prime}}^{Z}(\mathbb{T}, \beta)
$$

from (37). Hence, it is enough to show only (21-d). Suppose (21-d) does not hold. We obtain a function $f=f_{*}+H$ from $f_{*}$ and $H$. As in Case 21-1, $f$ fulfills all conditions in Lemma 21. This finishes Case 21-3.

By Cases 21-1, 21-2, and 21-3, we have finished the proof of Lemma 21.

Now we prove Case 14-3. Suppose $\alpha$ is a successor ordinal bigger than or equal to 3. By Lemma 21, we obtain a differentiable function satisfying Theorem 14 . So we let $\alpha$ be a countable limit ordinal. We take a sequence of ordinals $\left\langle\beta_{n}\right\rangle$ such that $3 \leq \beta_{n}<\beta_{n+1}$ and $\lim _{n \rightarrow \infty} \beta_{n}=\alpha$. As usual, we choose small enough $\varepsilon_{n}$ 's. 
For each $\varepsilon_{n}$, we obtain a differentiable function $f_{\beta_{n}}$ satisfying Lemma 21 . We then define $f$ as follows:

$$
f(x)= \begin{cases}1 / 2^{k+1} F_{k}(x) f_{\beta_{n}}\left(M_{k}\left(x-c_{k}\right)\right), & \text { if } x \in\left[a_{k}, b_{k}\right] ; \\ 0, & \text { otherwise. }\end{cases}
$$

We note that for any $\delta>0$,

$$
\partial_{f, \delta}^{K W}(\mathbb{T}, 1)=\partial_{f}^{K W}(\mathbb{T}, 1) \text { and }\left|\partial_{f}^{K W}(\mathbb{T}, 1)\right|_{C B}=\alpha+1 .
$$

By Remark $7,|f|_{K W}$ and $|f|_{Z}$ are less than or equal to $\alpha+1$. By Lemma 21 , it is easy to show that $|f|_{Z}=\alpha+1$ but $|f|_{D J}=2$. Therefore, Theorem K asserts the Kechris-Woodin rank of $f$ should be $\alpha+1$. This completes the proof of Case 14-3.

By Cases 14-1, 14-2, and 14-3, this completes the proof of Theorem 14.

\section{Conclusion}

We have seen that the Denjoy rank and the Zalcwasser rank are incomparable. We have one more theorem on this result. By combining Theorem 9, Theorem 14, and Theorem KW, we derive the following theorem:

Theorem 23. Let $\alpha, \beta$ and $\gamma$ be countable ordinals. Suppose $\alpha, \beta<\gamma$ and $\beta$ is a nonzero ordinal. Then there exists a function in $D(\mathbb{T})$ such that

$$
|f|_{Z}=\alpha+1,|f|_{D J}=\beta+1 \text { and }|f|_{K W}=\gamma .
$$

Proof of Theorem 23. We fix $\alpha, \beta$, and $\gamma$. By Theorem 9, Theorem 14, and Theorem $\mathrm{KW}$, we have $f_{1}$ and $f_{2}$ in $D(\mathbb{T})$ and $f_{3}$ in $b_{1} D(\mathbb{T})$ such that

(23-a) $f_{1}(0)=f_{1}^{\prime}(0)=f_{2}(0)=f_{2}^{\prime}(0)=f_{3}(0)=f_{3}^{\prime}(0)=0$;

(23-b) $\left|f_{1}\right|_{Z}=\left|f_{1}\right|_{K W}=\alpha+1$ and $\left|f_{1}\right|_{D J}=2$;

(23-c) $\left|f_{2}\right|_{D J}=\left|f_{2}\right|_{K W}=\beta+1$ and $\left|f_{1}\right|_{Z}=1$;

(23-d) $\left|f_{3}\right|_{K W}=\gamma$ and $\left|f_{3}\right|_{D J}=\left|f_{3}\right|_{Z}=1$.

We then define

$$
f(x)= \begin{cases}f_{1}(3 x), & \text { if } 0 \leq x \leq \pi \\ f_{2}(3(x-2 \pi / 3)), & \text { if } 2 \pi / 3 \leq x \leq 4 \pi / 3 \\ f_{3}(3(x-4 \pi / 3)), & \text { otherwise }\end{cases}
$$

Then $f$ is well-defined and differentiable by (23-a). By (23-b), (23-c), and (23-d), it is easy to see that $|f|_{Z}=\alpha+1,|f|_{D J}=\beta+1$ and $|f|_{K W}=\max \{\alpha+1, \beta+1, \gamma\}=$ $\gamma$.

This proves Theorem 23.

\section{ACKNOWLEDGMENT}

I wish to thank Professors A. Kechris and R. Jin, and S. Solecki for helpful conversations. I would like to show my appreciation to Professor Kaufman for inviting me to give a talk based on this paper. Finally, I thank the referee for many valuable remarks. 


\section{REFERENCES}

[AK] M. Ajtai and A. S. Kechris, The set of continuous functions with everywhere convergent Fourier series, Trans. Amer. Math. Soc. 302 (1987), 207-221. MR 89b:04005

[Br] A. M. Bruckner, Differentiation of real functions, Lecture Notes in Math., vol. 659, Springer-Verlag, Berlin and New York, 1978. MR 80h:26002

[GH] D. C. Gillespie and W. A. Hurwitz, On sequences of continuous functions having continuous limits, Trans. Amer. Math. Soc. 32 (1930), 527-543.

[Ka] Y. Katznelson, An introduction to harmonic analysis, 2nd ed., Dover, New York, 1976. MR 54:1097b

[Ke] A. Kechris, Classical descriptive set theory, Springer Verlag, New York, 1995. MR 96e: 03057

[Ki] H. Ki, The Kechris-Woodin rank is finer than the Zalcwasser rank, Trans. Amer. Math. Soc. 347 (1995), 4471-4484. MR 96b:04004

$[\mathrm{KW}]$ A. S. Kechris and W. H. Woodin, Ranks for differentiable functions, Mathematika 33 (1986), 252-278. MR 88d:03097

[Ma] S. Mazurkiewicz, Über die Menge der differenzierbaren Funktionen, Fund. Math. 27 (1936), $244-249$

[Mo] Y. N. Moschovakis, Descriptive set theory, North-Holland, Amsterdam, 1980. MR 82e:03002

[Ra] T. I. Ramsamujh, Three ordinal ranks for the set of differentiable functions, J. Math. Anal. and Appl. 158 (1991), 539-555. MR 92h:26010

[Za] A. Zalcwasser, Sur une propriété du champs des fonctions continues, Studia Math. 2 (1930), 63-67.

[Zy] A. Zygmund, Trigonometric series, 2nd ed., Cambridge Univ. Press, 1959. MR 21:6498

Department of Mathematics, Yonsei University, Seoul, 120-749, Korea

E-mail address: haseo@bubble.yonsei.ac.kr 